2. Ghincea CV, Anderson DA, Ikeno Y, Roda GF, Eldeiry M, Bronsert MR, et al. Utility of neuromonitoring in hypothermic circulatory arrest cases for early detection of stroke: listening through the noise. J Thorac Cardiovasc Surg. 2021;162:1035-45.e5.

3. Nwachuku EL, Balzer JR, Yabes JG, Habeych ME, Crammond D, Thirumala PD. Diagnostic value of somatosensory evoked potential changes during carotid endarterectomy: a systematic review and meta-analysis. JAMA Neurol. 2015;72: 73-80.

4. Cheung AT, Savino JS, Weiss SJ, Patterson T, Richards RM, Gardner TJ, et al. Detection of acute embolic stroke during mitral valve replacement using somatosensory evoked potential monitoring. Anesthesiology. 1995;83:208-10.

5. Stecker MM, Cheung AT, Patterson T, Savino JS, Weiss SJ, Richards RM, et al. Detection of stroke during cardiac operations with somatosensory evoked responses. J Thorac Cardiovasc Surg. 1996;112: 962-72.

6. Liu LY, Callahan B, Peterss S, Dumfarth L, Tranquilli M, Ziganshin BA, et al Neuromonitoring using motor and somatosensory evoked potentials in aortic surgery. J Card Surg. 2016;31:383-9.

7. Yoshitani K, Masui K, Kawaguchi M, Kawamata M, Kakinohana M, Kato S, et al Clinical utility of intraoperative motor-evoked potential monitoring to prevent postoperative spinal cord injury in thoracic and thoracoabdominal aneurysm repair: an audit of the Japanese Association of Spinal Cord Protection in Aortic Surgery Database. Anesth Analg. 2018;126:763-8.

8. Chung J, Ouzounian M, Lindsay T. Motor evoked potential monitoring during thoracoabdominal aortic surgery: useful or not? Anesth Analg. 2018;126: $741-2$.
See Article page 1035.

\section{Commentary: If the news is good, it is better that we know ... if the news is bad, it is better than we know fast}

\author{
Lisa Q. Rong, MD, ${ }^{\mathrm{a}}$ Luca P. Weltert, MD, ${ }^{\mathrm{b}}$ and \\ Mario F. L. Gaudino, MD
}

The risk of stroke in aortic arch (AA) surgery remains a serious concern, despite modern surgical techniques and strategies for cerebral protection. Brain perfusion during AA surgery faces embolic and global ischemic threats, from manipulation and crossclamping of the aorta to malperfusion due to incomplete protective perfusion during the circulatory arrest phase of the operation. Intraoperative diagnosis of stroke can be a challenge, and there are no current standard guidelines for intraoperative neuromonitoring (IONM).

Despite this, data from IONM may allow for early diagnosis of stroke and inform subsequent management of

\footnotetext{
From the a Department of Anesthesiology, Weill Cornell Medicine/New York Presbyterian Hospital, New York, NY; ${ }^{\mathrm{b}}$ Department of Cardiac Surgery, European Hospital, Rome, Italy; and ${ }^{\mathrm{c} D e p a r t m e n t}$ of Cardiothoracic Surgery, Weill Cornell Medicine/New York Presbyterian, New York, NY.

Disclosures: Lisa Q. Rong is supported in part by the Foundation for Anesthesia Research and Education Training Grant (FAER MTRG-CT-08-15-2018-Rong). All other authors have nothing to disclose with regard to commercial support.

Received for publication Feb 11, 2020; accepted for publication Feb 13, 2020; available ahead of print Feb 21, 2020.

Address for reprints: Mario F. L. Gaudino, MD, Department of Cardio-thoracic Surgery, Weill Cornell Medicine, 525 East 68th St, New York, NY 10065 (E-mail: mfg9004@med.cornell.edu).

J Thorac Cardiovasc Surg 2021;162:1047-8

0022-5223/\$36.00

Copyright (c) 2020 by The American Association for Thoracic Surgery

https://doi.org/10.1016/j.jtcvs.2020.02.058
}

Check for updates

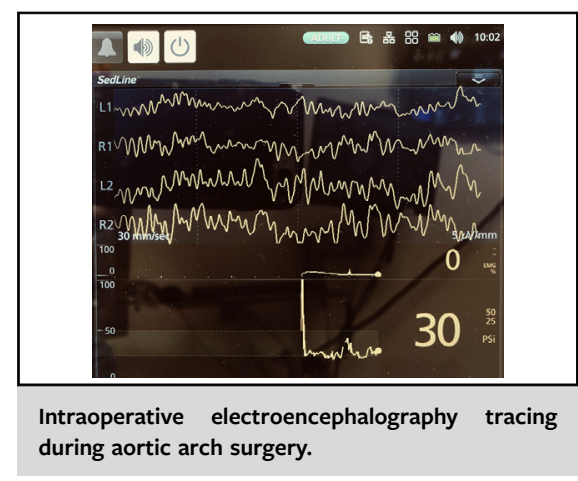

CENTRAL MESSAGE

Intraoperative neuromonitoring can provide early diagnosis and intervention for stroke and provide reassurance that cerebral perfusion is adequate during aortic arch surgery.

the patient. Although the operation may not be aborted, interventions such as optimizing oxygen delivery through increasing mean arterial blood pressure and hematocrit and by decreasing cerebral metabolic rate may decrease sequelae of cerebral hypoperfusion. In addition, in the cases of embolic stroke, early diagnosis allows for the stroke team to be on standby for the possibility of emergent imaging and intervention after the operation is complete. IONM strategies used include electroencephalography (EEG) and evoked potential measurements, transcranial Doppler, and near-infrared spectroscopy (NIRS).

NIRS is most common technique used to diagnose and treat cerebral hypoperfusion in AA surgery. Although 
evidence suggests that NIRS assists during cannulation positioning for cerebral perfusion during aortic surgery, there are insufficient data demonstrating the ability of NIRS in decreasing risk of stroke or cognitive dysfunction in general. ${ }^{1}$ Similarly, while NIRS has been used to determine individual patient cerebral autoregulation curves during $\mathrm{CPB},{ }^{2}$ this has not yet been shown to improve neurologic outcomes. ${ }^{3}$

EEG, motor-evoked potentials (MEPs), and somatosensory-evoked potential (SSEP) changes such as slowing, decreased amplitude, increased latency, and development of asymmetric signals may suggest ischemia intraoperatively outside the period of hypothermic circulatory arrest. However, the need for experienced personnel and high rate of false-negative alerts, due to similar patterns caused by anesthetic agents and hypothermia mimicking ischemia, may limit the use of EEG, MEP, and SSEP clinically. Processed EEG is more popular because of the ease of interpretation but evidence is inconclusive on its utility to detect ischemia. ${ }^{4-7}$

Ghincea and colleagues ${ }^{8}$ address an important deficit in the literature regarding the utility of using this type of neuromonitoring 223 of 365 patients undergoing arch surgery over an 8-year period. In their experience, the sensitivity of using MEPs, SSEPs, and EEG was $75 \%$, with a specificity of $88.5 \%$, positive predictive value (PPV) of $27.3 \%$, and a negative predictive value of $97.4 \%$ with a significant increase in PPV, negative predictive value, and specificity when excluding patients in whom IONM abnormalities returned to baseline at the end of the operation. $^{3}$ These are promising results; intraoperative monitoring abnormalities, although they have limitations, can be used to guide relatively benign and low-cost interventions that will likely help and not harm the patient. Further cost and resource use will be limited until after the operation, where the test has a significant greater PPV and specificity. The absence of alerts has the benefit of reassuring both the surgeon and cardiac anesthesiologist that there is adequate cerebral perfusion. In the age of embracing technology and culture of more information is better, IONM described by Ghincea and colleagues may be an attractive option for surgeons, patients, and cardiac anesthesiologists in the perioperative period. The increased cost of monitoring may be worth the peace of mind. In the rare cases of stroke, neuromonitoring may provide opportunities for early intervention, mitigate the extent of ischemia, and improve clinical outcomes, which we consider as priceless.

\section{References}

1. Zheng F, Sheinberg R, Yee M-S, Ono M, Zheng Y, Hogue CW. Cerebral nearinfrared spectroscopy monitoring and neurologic outcomes in adult cardiac surgery patients: a systematic review. Anesth Analg. 2013;116:663-76.

2. Brady K, Joshi B, Zweifel C, Smielewski P, Czosnyka M, Easley RB, et al. Real-time continuous monitoring of cerebral blood flow autoregulation using near-infrared spectroscopy in patients undergoing cardiopulmonary bypass. Stroke. 2010;41:1951-6.

3. Brown CH, Neufeld KJ, Tian J, Probert J, LaFlam A, Max L, et al. Effect of targeting mean arterial pressure during cardiopulmonary bypass by monitoring cerebral autoregulation on postsurgical delirium among older patients: a nested randomized clinical trial. JAMA Surg. May 22, 2019 [Epub ahead of print].

4. Bonhomme V, Desiron Q, Lemineur T, Brichant JF, Dewandre P-Y, Hans P. Bispectral index profile during carotid cross clamping. J Neurosurg Anesthesiol. 2007;19:49-55.

5. Estruch-Pérez MJ, Ausina-Aguilar A, Barberá-Alacreu M, Sánchez-Morillo J, Solaz-Roldán C, Morales-Suárez-Varela MM. Bispectral index changes in carotid surgery. Ann Vasc Surg. 2010;24:393-9.

6. Estruch-Pérez MJ, Barberá-Alacreu M, Ausina-Aguilar A, Soliveres-Ripoll J, Solaz-Roldán C, Morales-Suárez-Varela MM. Bispectral index variations in patients with neurological deficits during awake carotid endarterectomy. Eur J Anaesthesiol. 2010;27:359-63.

7. Deogaonkar A, Vivar R, Bullock RE, Price K, Chambers I, Mendelow AD. Bispectral index monitoring may not reliably indicate cerebral ischaemia during awake carotid endarterectomy. Br J Anaesth. 2005;94:800-4.

8. Ghincea CV, Anderson DA, Ikeno Y, Roda GF, Eldeiry M, Bronsert M, et al. Utility of neuromonitoring in hypothermic circulatory arrest cases for early detection of stroke: listening through the noise. J Thorac Cardiovasc Surg. 2021;162:1035-45.e5. 